EPJ Web of Conferences 53, 01014 (2013)

DOI: $10.1051 /$ epjconf/20135301014

(C) Owned by the authors, published by EDP Sciences, 2013

\title{
Space-based observation of the extensive airshowers
}

\author{
T. Ebisuzaki
}

RIKEN Advanced Science Institute, 2-1 Hiroswa, Wako 351-0198, Japan

\begin{abstract}
Space based observations of extensive air showers constitute the next experimental challenge for the study of the universe at extreme energy. Space observation will allow a "quantum jump" in the observational area available to detect the UV light tracks produced by particles with energies higher than $10^{20} \mathrm{eV}$. These are thought to reach the Earth almost undeflected by the cosmic magnetic field. This new technique will contribute to establish the new field of astronomy and astrophysics performed with charged particles and neutrinos at the highest energies. This idea was created by the incredible efforts of three outstanding comic ray physicists: John Linsley, Livio Scarsi, and Yoshiyuki Takahashi. This challenging technique has four significant merits in comparison with ground-based observations: 1) Very large observational area, 2) Well constrained distances of the showers, 3) Clear and stable atmospheric transmission in the above half troposphere, 4) Uniform Exposure across both the north and south skies. Four proposed and planned missions constitute the roadmap of the community: TUS, JEM-EUSO, KLPVE, and Super-EUSO will contribute step-by-step to establish this challenging field of research.
\end{abstract}

\section{INTRODUCTION}

Space based observation of the extensive air showers (EAS) constitutes the next generation technology for the study of the extreme universe. This new and challenging technique allows a quantum jump in the observational area used to detect UV tracks associated to EAS produced by particles with energies higher than $10^{20} \mathrm{eV}$. These particles are thought to reach the Earth almost not deflected by the cosmic magnetic fields. This new technique will contribute to establish the new field of astronomy and astrophysics performed with charged particles and neutrinos at the highest energies. The aim of the present paper is to present the concept of space based observation of EAS. We start from a history of this novel concept in section 2. In section 3, we discuss the main differences between the ground and the space based observational technique. In section 4, all proposed and planned missions are presented. Those are TUS, JEM-EUSO, KLPVE, and S-EUSO. In Section 5 we present the summary and conclusions.

\section{HISTORY OF SPACE BASED CONCEPT FOR AIRSHOWER OBSERVATION}

The concept of space based observations of EAS was developed by the joint efforts of "three giants" in the field of highest energy cosmic rays: John Linsley, Livio Scarsi, and Yoshiyuki Takahashi. This novel idea can be traced back to SOCRAS, the Satellite Observatory of Cosmic Ray Showers, suggested by John Linsley in the late 1970's, when he explicitly suggested SOCRAS as one of the projects included in the Field Committee Report of NASA "Call for Projects and Ideas in High Energy Astrophysics for the 1980s". The key idea at the base of SOCRAS concept was very clear: to observe, by means of spacebased devices, looking at nadir during the night, the fluorescence light produced by an EAS proceeding in the atmosphere. Linsley's original concept was based on a $38 \mathrm{~m}$ diameter mirror to monitor a circular field of about $100 \mathrm{~km}$ in diameter, corresponding to an area of $10^{4} \mathrm{~km}^{2}$ and an air mass of $10^{11}$ tons, from

This is an Open Access article distributed under the terms of the Creative Commons Attribution License 2.0, which permits unrestricted use, distribution, and reproduction in any medium, provided the original work is properly cited. 


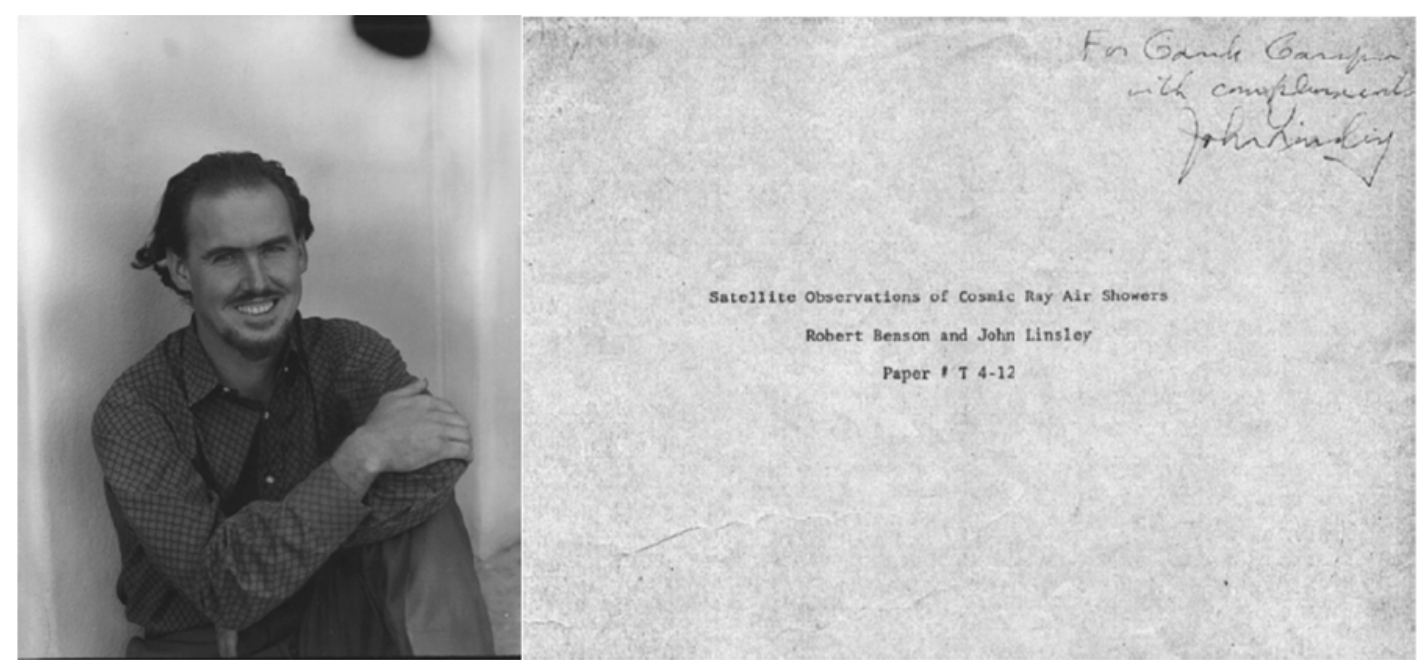

Figure 1. Left: John Linsley, who first suggested space based observations of extreme energy cosmic rays with SOCRAS: the Satellite Observatory of Cosmic Ray Showers; Right: the cover page of the preprint in which Benson and Linsley (1981) presented SOCRAS [1].

a circular orbit at about 500-600 km above the surface [1]. The idea was visionary but unfortunately not feasible with the imaging and space technology of the 80's.

The key breakthrough in the imaging technology was suggested in 1995 by Yoshiyuki Takahashi, who proposed the use of light-weight Fresnel optics to enlarge Field of View to \pm 30 degree keeping a reasonable size [2]. Takahashi named the new approach MASS: Maximum-energy Auger (Air)-Shower Satellite. It's interesting to learn about those hectic months from the words of John Linsley.

On 15, May 1995, my wife Paola telephoned me in Palermo that Yoshiyuki Takahashi was trying to get in touch with me from Marshall Space Flight Centre. His message was "I have written a paper about a maximum-energy Air shower Observing Satellite. The technology and neutrino detection capability relate to John's original ideal of 1979. I would like to send my text to John, and talk with him." As soon as we have talked, I told Livio Scarsi what happened. "It sounds as if might be fun," he said. "But it will be hard for me to explain "MASS" to the Italian Space Agency. We should call it something more general." After a few tries we came up with "Airwatch," short for "Space Watch" (John Linsley, [3]).

Takahashi's breakthroughs were the reduced aperture and the enlargement of the field of view of the optical system. The enlargement in the field of view increased the expected statistics, while the reduction in the aperture made the instrument feasible as a space instrument. Linsley considered the Airwatch approach as the fourth breakthrough in the search of the end of the cosmic ray energy spectrum [3]. In a very short time, Takahashi organised a seminal workshop that took place in Huntsville in August 1995. By the following spring, a group formed at the Godadrd Space Flight Center/NASA prepared a proposal for the OWL mission, a pair of Orbiting Wide angle Light concentrators [4]. The OWL mission study proposal was accepted in 1996 and included in NASA's SEU Mid-term strategic Plan (2010's).

Meanwhile and in parallel, the first Airwatch symposium, in which a number of contributors from Italy and Russia participated, was held in Catania in 1996. The efforts in Europe evolved into the concept of EUSO: the Extreme Universe Space Observatory, under the leadership of Livio Scarsi. The EUSO proposal submitted under the PI-ship of Livio Scarsi to the ESA call for the F2/F3 missions was selected and re-oriented for an accommodation study onto the International Space Station (ISS) in January 2000 [5]. EUSO with light-weight Fresnel lenses was designed to achieve a wide field of view of 60 degrees. Immediately after a successful one-year long accommodation study, EUSO was approved for a Phase-A study as an external payload for the European Columbus module of the ISS in 


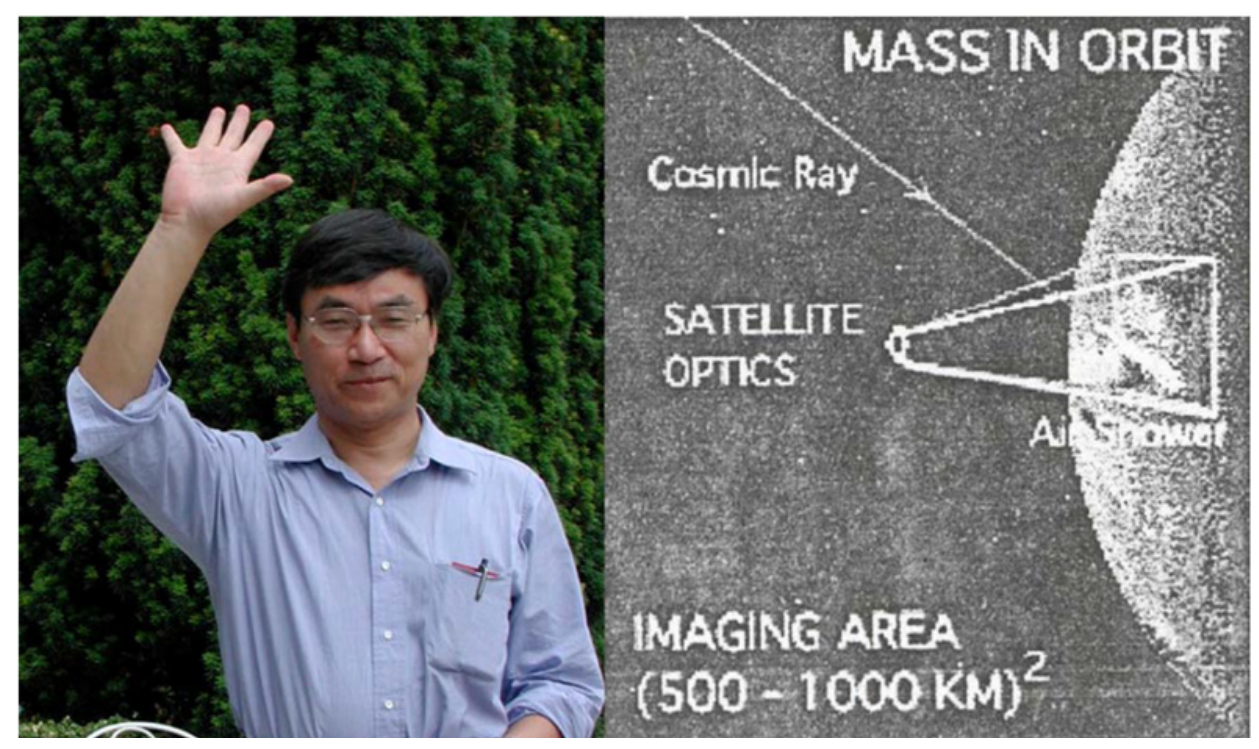

Figure 2. Left: Yoshiyuki Takahashi; Right: the concept of MASS, the Maximum-Energy Auger (Air)-Shower Satellite.

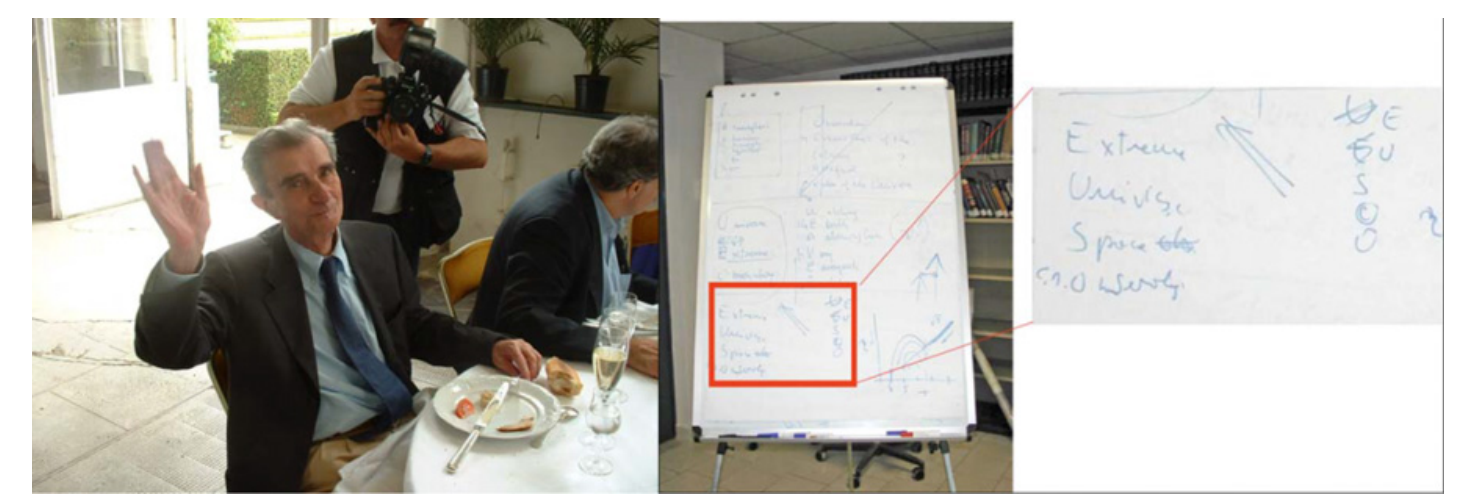

Figure 3. Left: Professor Livio Scarsi, enjyoing lunch at a restaurant in Palermo; Right: how the EUSO acronym was obtained.

March 2001. The Phase A study was successfully concluded in March 2004 [6, 7]. However, because of programmatic and financial problems at ESA and in some leading European countries, and because of the Columbia disaster, the start of phase-B was postponed indefinitely. The Japanese and the U.S. teams, under the strong leadership of Yoshiyuki Takahashi, promptly re-defined EUSO as a mission attached to the Japanese Experiment Module/Exposure Facility (JEM/EF) of the ISS. The mission was renamed as JEM-EUSO and started all necessary studies targeting launch in 2013 in the framework of the second phase utilization of JEM/EF utilization [8-12]. The Kick-off meeting of the renewed EUSO mission was held at RIKEN in 2006.

During the same period, researchers in Russia planned other missions based on Fresnel mirrors, developed by their own technology. These efforts materialised into the TUS [13-17] and KLPVE $[18,19]$ missions, which we will describe later in the present paper. 


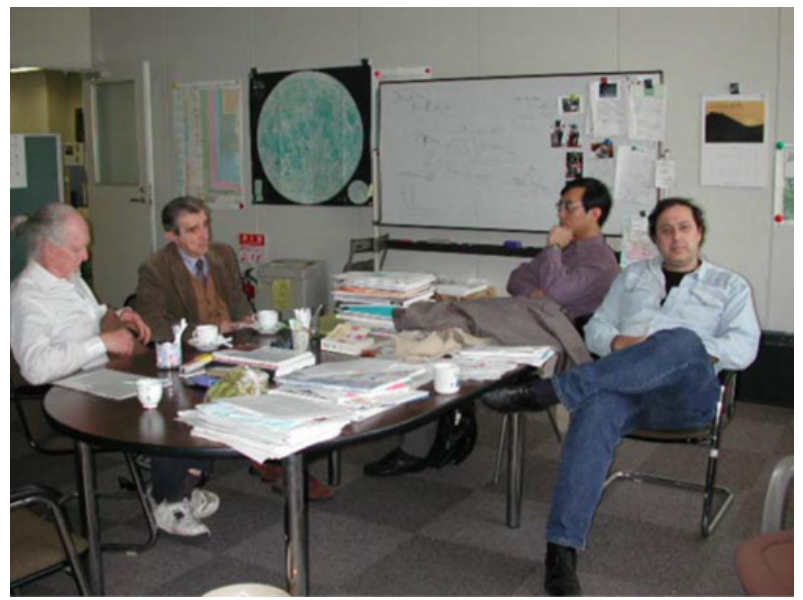

Figure 4. From left to right, John Linsley, Livio Scarsi, and Yoshiyuki Takahashi with Osvaldo Catalano at RIKEN in 2001 .

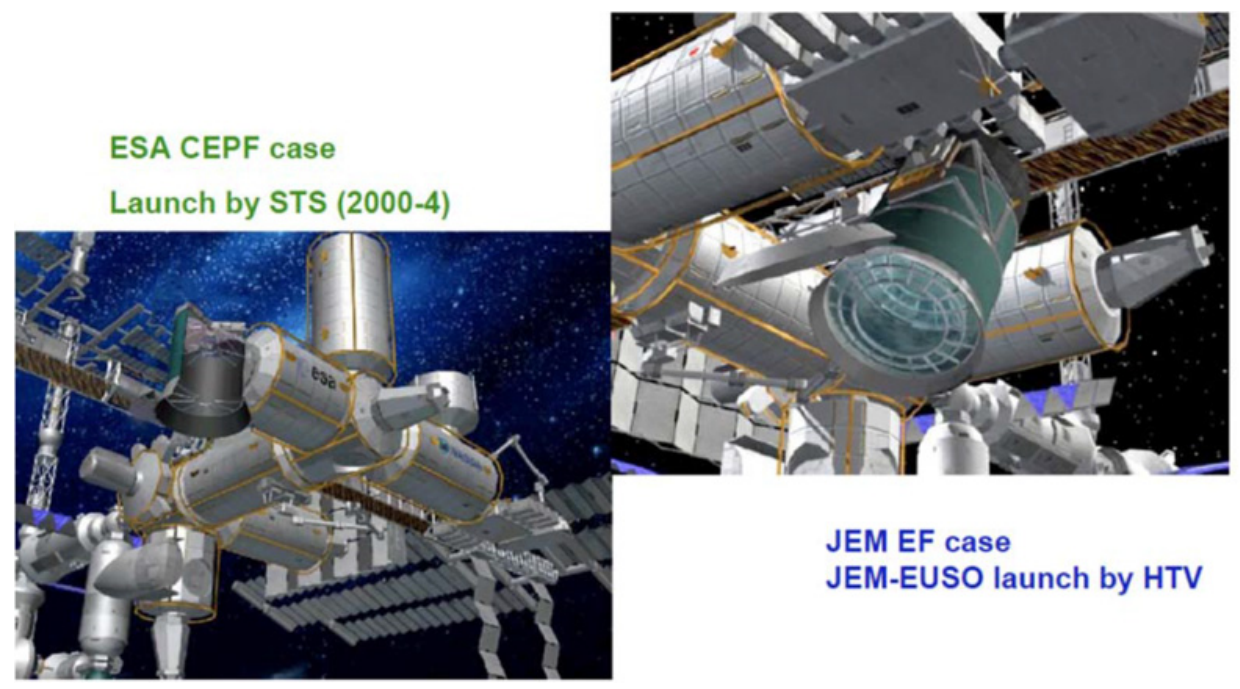

Figure 5. Artistic drawings of ESA-EUSO (left) and JEM-EUSO (right).

\section{FOUR MERITS OF LOOKING-DOWN COMPARED WITH LOOKING-UP}

The space-based approach, to observe from above and looking downward the EAS generated in the Earth's atmosphere has four significant merits compared with the conventional looking-up approach from the ground: 1) Very large observational area, 2) Well constrained distances toward showers, 3) Clear and stable atmospheric transmission in the above half troposphere, 4) Uniform Exposure across both north and south skies.

\subsection{Very large observational area}

The most relevant merit of the space based observation is the very large area that can be monitored from space. In the case of JEM-EUSO, for example, the instantaneous observational area is $2 \times 10^{5} \mathrm{~km}^{2}$ in 

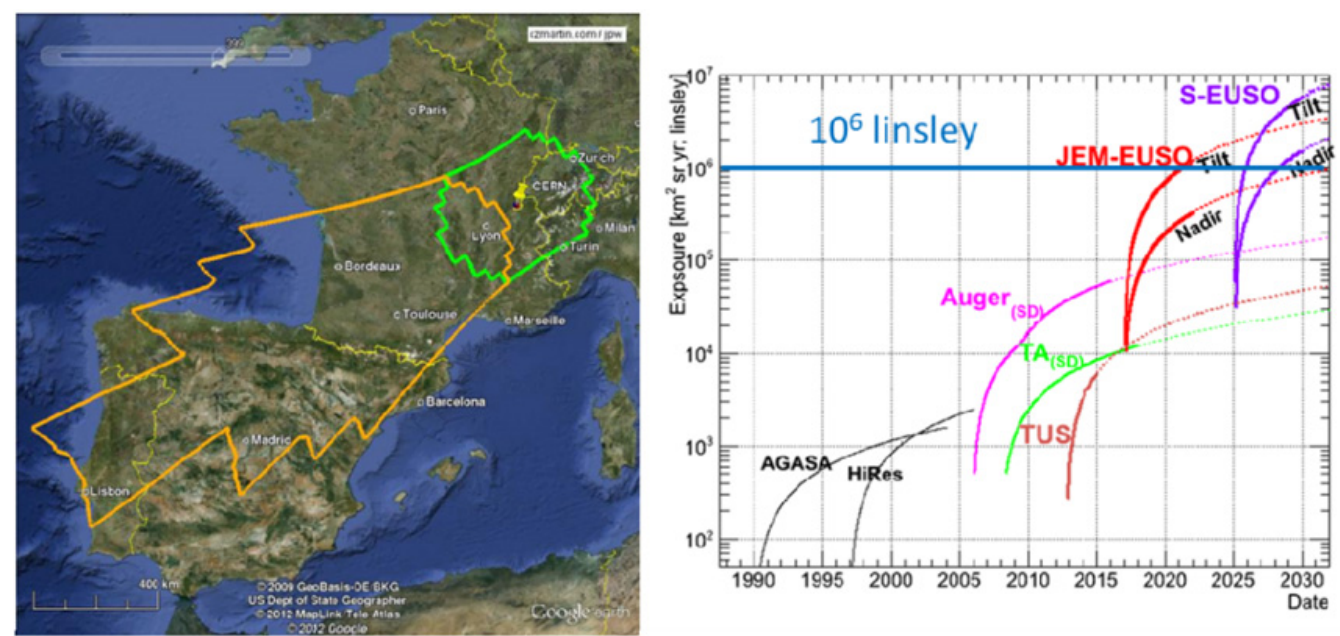

Figure 6. Left: footprint of the field of view of the JEM-EUSO telescope, projected to west Europe: Nadir mode (Green) and Tilted mode (Orange); Right: expected time evolution of the exposure. Space-based missions JEMEUSO (and KLPVE), and Super-EUSO can achieve the critical threshold of $10^{6} \mathrm{~km}^{2}$ str yr.

\section{working principle of Space based Observation}

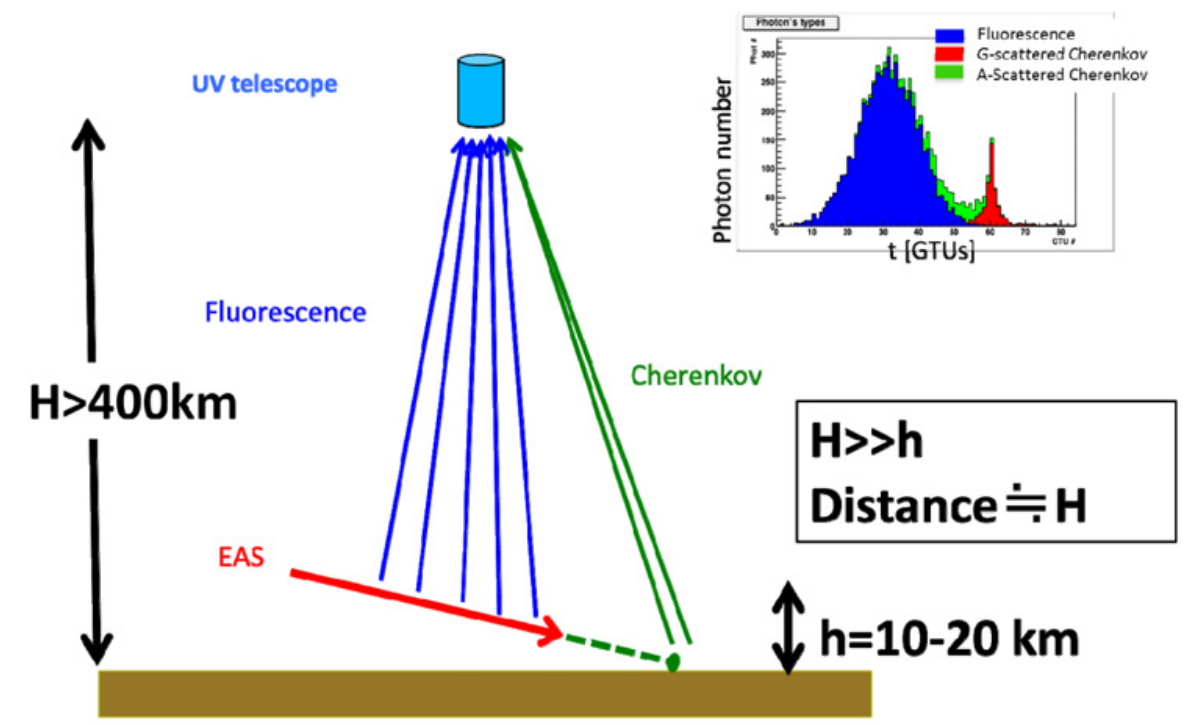

Figure 7. The geometry of detection which shows the small proximity effect.

nadir mode and can reach $7 \times 10^{5} \mathrm{~km}^{2}$ in tilted mode. These are almost two orders of magnitude larger than the largest ground based observatories $\left(\sim 3 \times 10^{3} \mathrm{~km}^{2}\right.$ in the case of the Pierre Auger Observatory). Such a geometrical area allow us to reach the impressive exposure of $10^{6} \mathrm{~km}^{2}$ str y $\left(10^{6}\right.$ Linsley), considered critical for astronomy and astrophysics with charged particles and for the detection of neutrinos above $10^{20} \mathrm{eV}$. 


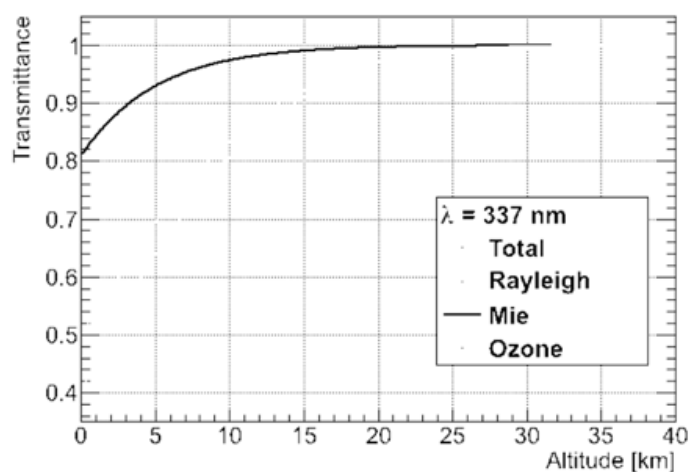

Figure 8. Transmission of the upper half of the atmosphere is dominated by the Rayleigh scattering, while Mie scattering due to dust and aerosol particles is significant in the lower half.

\subsection{Well constrained distances toward showers}

The distance between the detector and the EAS is well constrained in the case of space observations since and the part of the atmosphere where showers are located is small $(10-20 \mathrm{~km})$ compared with the altitude (300-400 km) of the orbit (Fig. 8). On the other hand, in the ground-based observation, stereo or hybrid observations are necessary to determine the distance to the air showers.

\subsection{Clear and stable atmospheric transmission in the above half troposphere}

The propagation of the UV light in the upper half of the atmosphere is dominated by Rayleigh scattering, while Mie scattering due to dust particles and aerosols is significant only in the lower half of the atmosphere in clear skies (Fig. 8). While the effect of Rayleigh scattering does not change with time, the Mie scattering drastically depends on the surface conditions and can introduce a significant error in the estimate of the energy of the events.

Distributions of cloud top height and shower maximum height are shown in Fig. 9. Most of the clouds are located below $3 \mathrm{~km}$, while most EAS reach their maximum above $3 \mathrm{~km}$. We can observe showers even in cloudy sky conditions, if the cloud top is as low as $3 \mathrm{~km}$ (Fig. 9).

\subsection{Uniform exposure across the north and south skies}

The exposure of the space based mission can be almost uniform across the entire sky, as can be seen in Figure 11 for the case of the JEM-EUSO mission. In contrast, the ground observatory can observe only half spheres of the sky. The uniform exposure is essentially important for global arrival directional analysis.

\section{PROPOSED AND PLANNED MISSIONS}

According to the message which Livio Scarsi, PI of the EUSO mission, sent to the EUSO collaboration on January 2006, a step-by-step approach is necessary to realise space-based observation of extreme energy particles: 1) TUS in short-term, 2) JEM-EUSO in medium term, and 3) ESA Cosmic Vision (Super-EUSO) in medium-long term. This has been indeed the roadmap of the community. Four missions, TUS, JEM-EUSO, KLPVE, and S-EUSO have been planned and proposed. 


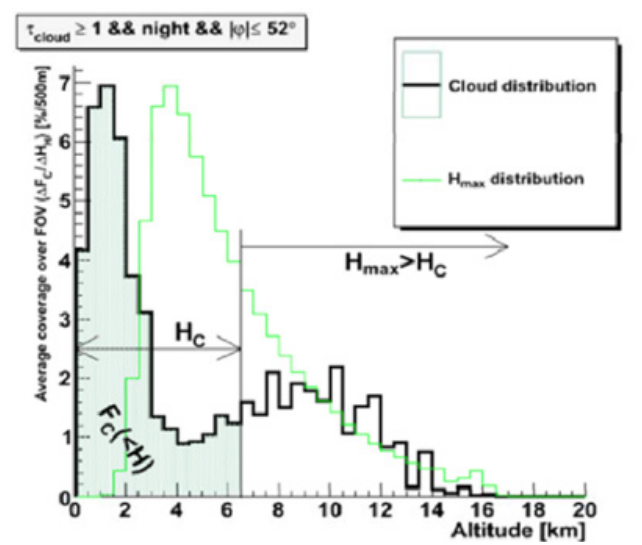

\begin{tabular}{|c|c|c|c|c|}
\hline $\begin{array}{l}\text { Clear sky } \sim 29 \% \\
\text { Green band } \sim 60\end{array}$ & & \multicolumn{2}{|c|}{ Cloud top } & \multirow[b]{2}{*}{$>10 \mathrm{~km}$} \\
\hline \multirow[b]{2}{*}{$O D>2$} & $<3 \mathrm{~km}$ & 3-7 km & $7-10 \mathrm{~km}$ & \\
\hline & 17.2 & 5.2 & 6.4 & 6.1 \\
\hline OD:1-2 & 5.9 & 2.9 & 3.5 & 3.1 \\
\hline OD:0.1-1 & 6.4 & 2.4 & 3.7 & 6.8 \\
\hline$O D<0.1$ & 29.2 & $<0.1$ & $<0.1$ & 1.2 \\
\hline
\end{tabular}

Figure 9. Left: the distribution of cloud top height and the altitude of the shower max. Most of the clouds locate bellow $3 \mathrm{~km}$, while most of the airshower reaches to their maximum above $3 \mathrm{~km}$; Right: statistics of clouds in top height and optical depth. We can observe airshowers even cloudy part of the Earth, if their heights are low enough (Green).
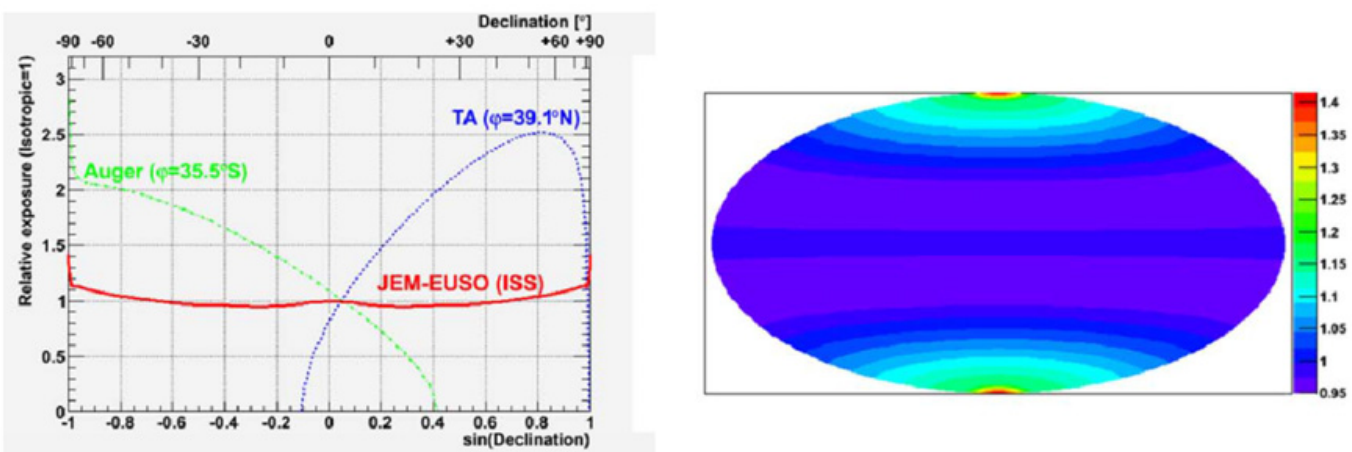

Figure 10. Left: the exposure of the JEM-EUSO mission is almost uniform across the north and south hemispeher of the sky because of the inclination (51.6 degree) of the ISS orbit in contrast to the ground observatory, which can observe only half of the sky; Right: expected exposure map of the JEM-EUSO mission.

\subsection{TUS}

TUS (Track UV Setup) is designed to be attached to the Lomonosov satellite, planned to be launched, to mark the 300th birthday anniversary of the Great Russian scientist Mikhail Lomonosov, in 2012 or 2013 by the Skobeltsyn Institute of Nuclear Physics of the Lomonosov Moscow State University, Russia [13-17]. TUS is the scientific pathfinder for space-based observation of EAS and for the global surveillance of the background UV level. It also recognised as a technical pathfinder for the JEM-EUSO mission: PMTs and electronics will be launched for space qualifications [20]. The UFFO (Ultra-Fast Flash Observatory) pathfinder, a Korean mission for gamma-ray burst, is also part of the payload of Lomonosov satellite [21]. The UVAT (UFFO Burst Alert and Trigger telescope) uses the PMT and electronics planned to be used in the JEM-EUSO mission.

\subsection{JEM-EUSO}

JEM-EUSO, as discussed above, is the "direct successor" of EUSO [8-12]. With respect to the ESA's EUSO mission concept, JEM-EUSO reduces the threshold energy down to $\sim 3 \times 10^{19} \mathrm{eV}$ and increases 


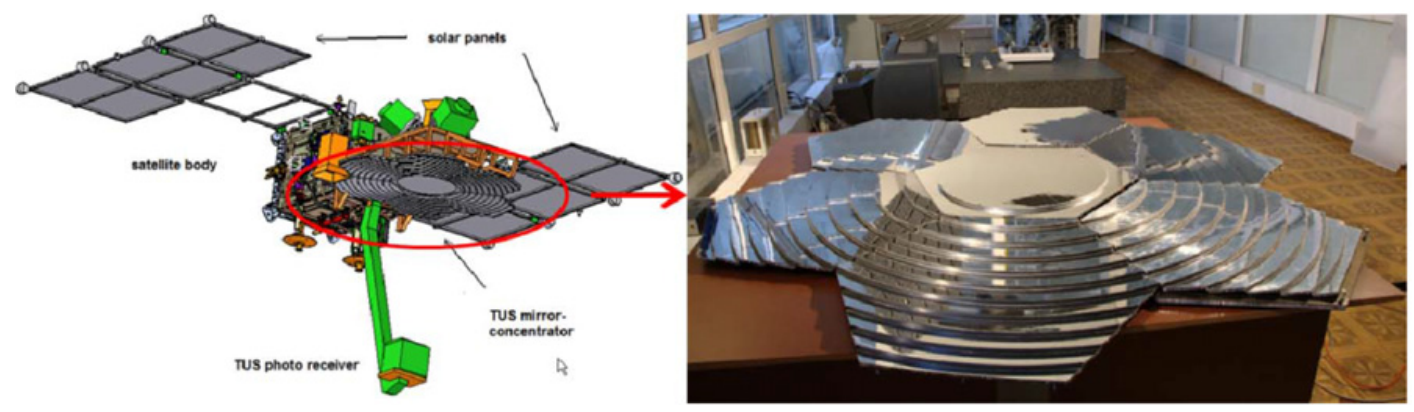

Figure 11. Left: TUS mission onboard Lomonosov statellite; Right: Fresnel mirror of the TUS mission.

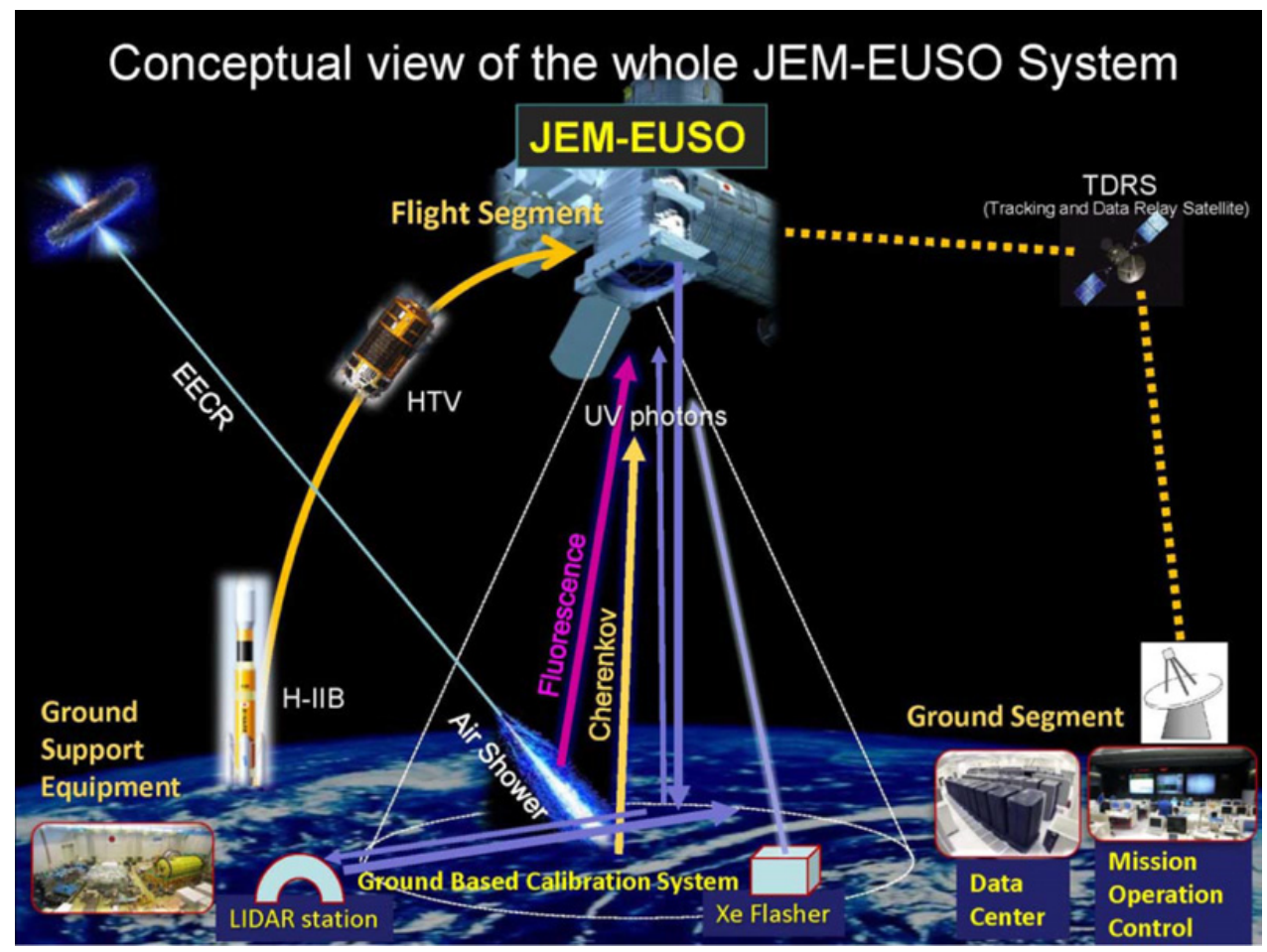

Figure 12. Concept of the JEM-EUSO mission.

the effective area by means of advances in technology and taking advantage of specific features of the $\mathrm{JEM} / \mathrm{EF}$ module. The reduction in the threshold energy is realized by using 1) a new lens material and an improved optical design, 2) detectors with higher quantum efficiency, and 3) an improved algorithm for event trigger. The increase in effective area is realized by inclining the telescope from nadir (socalled tilted mode). Although the threshold energy becomes higher, since the mean distance to EAS and atmospheric absorption both increase, in the $10^{20} \mathrm{eV}$ range, JEM-EUSO will be still full efficient. First few years of the mission lifetime will be devoted to observe lower energy region in nadir mode and then later to observe high energy region by tilted mode.

The JEM-EUSO telescope can reconstruct the incoming direction of the EECRs with accuracy better than a few degrees. Its observational aperture of the ground area is a circle with $250 \mathrm{~km}$ radius, and its atmospheric volume above it, with a $60^{\circ} \mathrm{FoV}$, is $\sim 1$ Tera-ton or more. JEM-EUSO, planned for the 


\section{UHECR 2012}
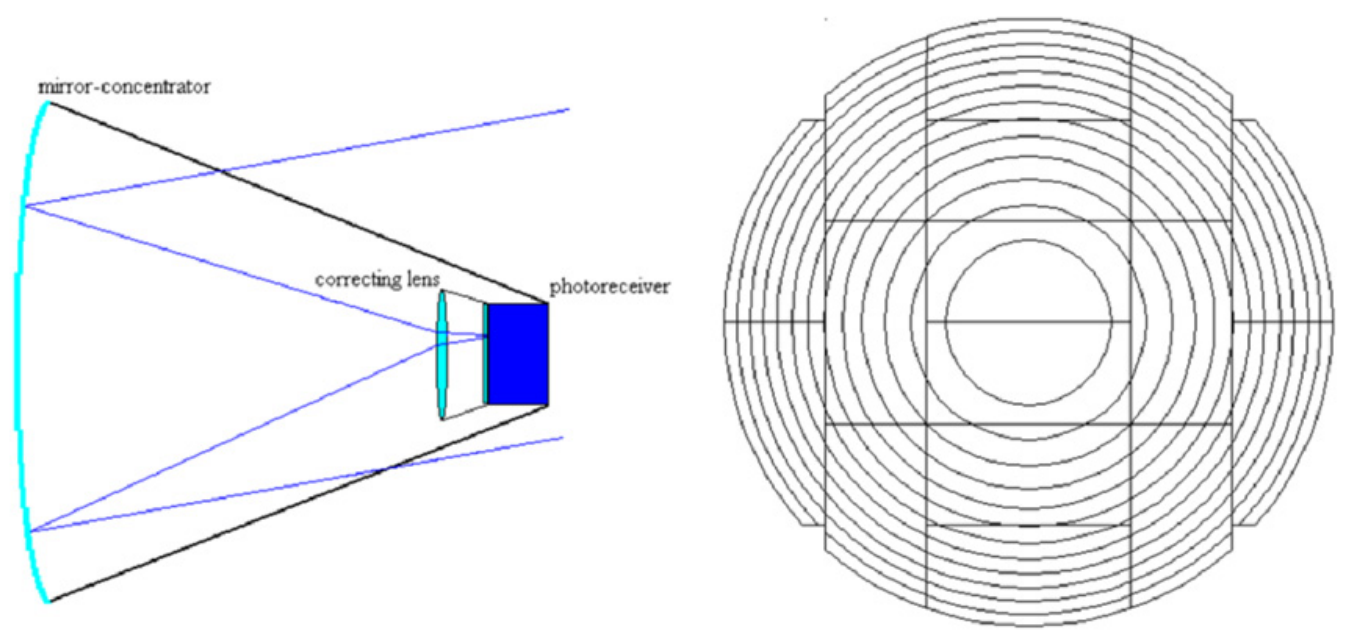

Figure 13. Left: KLYPVE telescope; Right: Fresnell mirror ( $8 \mathrm{~m}$ in diameter) of KLYPVE, segmented to sixteen pieces.

Table 1. Comparisons of four space-based missions.

\begin{tabular}{|c|c|c|c|c|c|c|}
\hline & $\begin{array}{l}\text { Optics } \\
\text { Aperture } \\
{\left[\mathrm{m}^{2}\right]}\end{array}$ & FOV & $\begin{array}{l}\text { Pixel } \\
\text { side }\end{array}$ & $\begin{array}{c}\text { Orbit } \\
\text { altitude } \\
{\left[\mathrm{km}^{2}\right]}\end{array}$ & $\begin{array}{l}\text { Geom. } \\
\text { aperture } \\
{\left[\mathrm{km}^{2} \mathrm{sr}\right]}\end{array}$ & $\begin{array}{c}\text { Annual } \\
\text { exposure } \\
{\left[\text { linsley } \mathrm{yr}^{-1} \text { ] }\right.}\end{array}$ \\
\hline $\begin{array}{l}\text { TUS } \\
(2012-)\end{array}$ & 1.8 & $9^{\circ} \times 9^{\circ}$ & $0.6^{\circ}$ & 500 & $2.0 \times 10^{4}$ & 2,700 \\
\hline $\begin{array}{l}\text { JEM- } \\
\text { EUSO } \\
(2017-)\end{array}$ & $\begin{array}{l}60^{\circ} \\
4.5\end{array}$ & $\begin{array}{c}\phi \times 48^{\circ} \\
\left(40^{\circ} \phi\right)\end{array}$ & $0.07^{\circ}$ & 400 & $\begin{array}{c}4.0 \times 10^{5} \\
\left(5.5 \times 10^{4}\right)\end{array}$ & $\begin{array}{l}60,000 \\
(7000)\end{array}$ \\
\hline $\begin{array}{l}\text { KLYPVE } \\
(2017-)\end{array}$ & 10 & $12^{\circ} \times 12^{\circ}$ & $<0.2^{\circ}$ & 400 & $2.2 \times 10^{4}$ & 3,000 \\
\hline $\begin{array}{l}\text { S-EUSO } \\
(2025-)\end{array}$ & 38 & $50^{\circ} \phi$ & $0.04^{\circ}$ & $\sim 900$ & $2.0 \times 10^{6}$ & 300,000 \\
\hline
\end{tabular}

JEM/EF of ISS, will be launched in the JFY 2016 by H2B rocket and conveyed to ISS by HTV (H-II transfer Vehicle; Fig. 12).

\subsection{KLYPVE}

KLYPVE is the science mission for EAS observation planned by SINP-MSU/ROSCOSMOS [18, 19]. It will be hosted onboard to Russian module of ISS. A Fresnel mirror with a diameter of four meters is segmented into sixteen pieces and assembled by an astronaut on-orbit (Fig. 13).

\subsection{Super-EUSO}

Super-EUSO is the scientific mission for the observation of charged particles and neutrinos in the energy above $10^{20} \mathrm{eV}$ [22]. It will have a photon corrector mirror of $8 \mathrm{~m}$ and field of view larger than 50 degrees. It was proposed as a free-flyer mission in the ESA Cosmic-vision in 2007. In a different version, SuperEUSO could be realised in the framework of space factory concept; in other words, assembled and tuned-up on ISS in orbit and released from there as a free-flyer [23, 24]. 

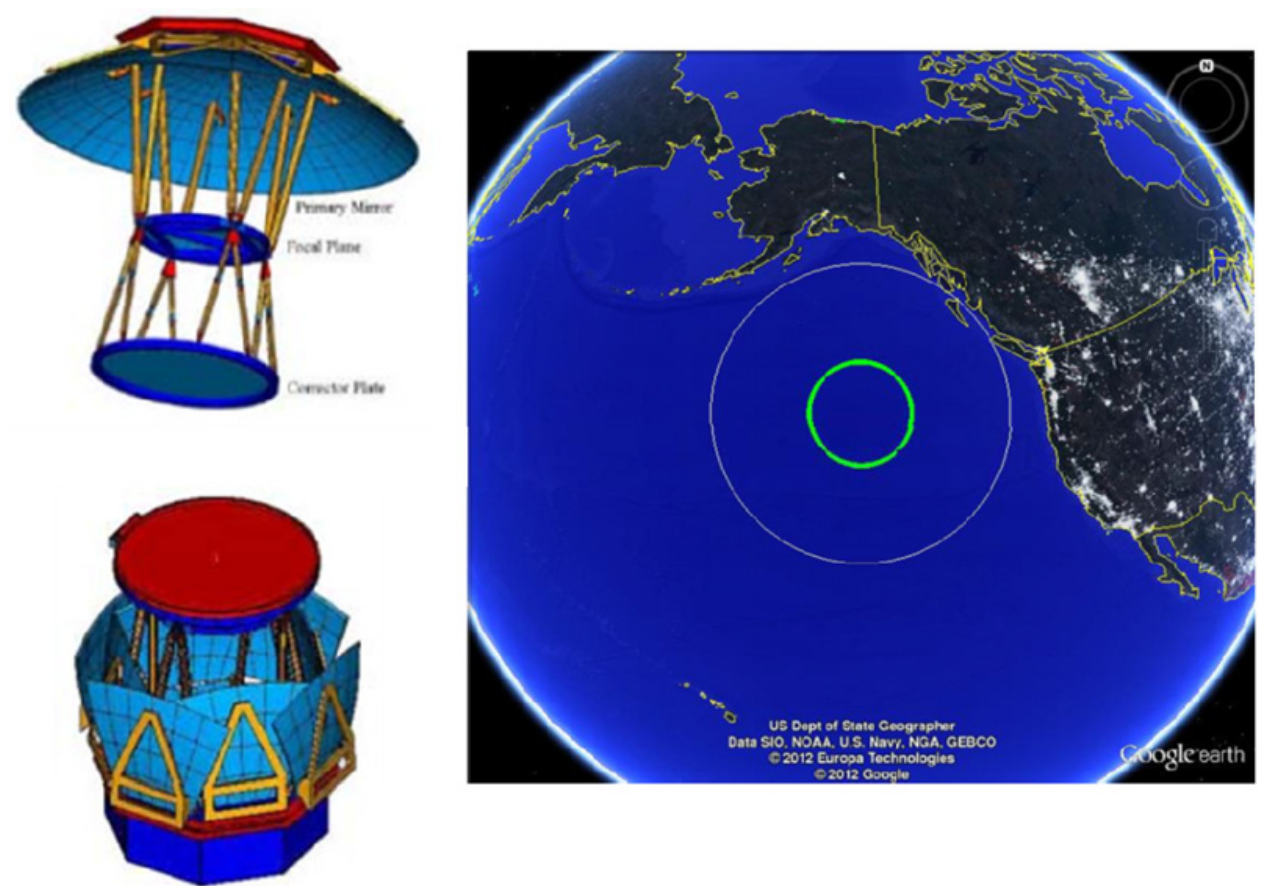

Figure 14. Concept of Super-EUSO; Upper left: observational configuration; Lower left: folded configuration for launch; Right: the field of view of Super-EUSO projected to Pacific Ocean.

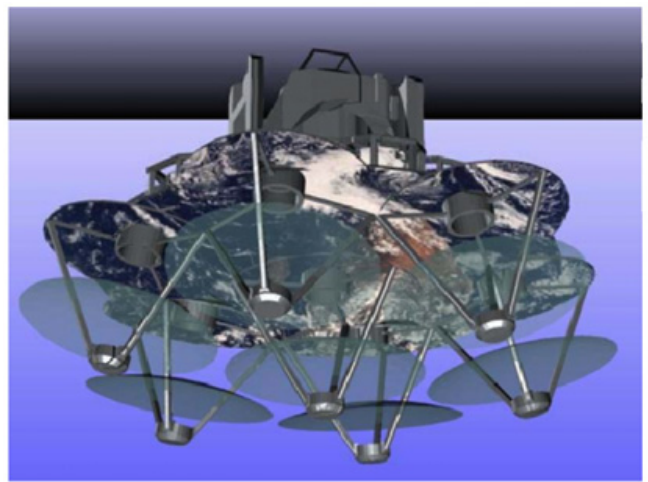

\section{Multi-units and/or multi-fleet $2 \times 3$ makes 6 times}

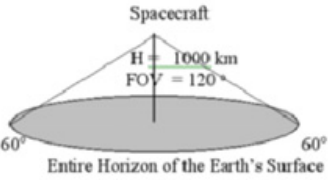

(a) Side View

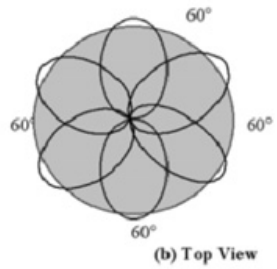

Figure 15. Left: artistic Drawing of Multi-OWL; Right: concept of Multi-OWL.

\subsection{AND BEYOND}

The comparison of the four missions is given in Table 1. The roadmap shows the exposure of $10^{6} \mathrm{~km}^{2}$ str $\mathrm{yr}$, is well within reach in the next decade. This is considered to be the critical value for astronomy and astrophysics through charged particles and the neutrino detection in highest energy region $\left(5 \times 10^{19} \mathrm{eV}\right)$. However, the roadmap could be extended. Yoshiyuki Takahashi proposed even a Multi-OWL concept [24] for even large observational area: In Multi-Owl mission, six or seven telescopes join together to enlarge the observational still by factor of six compared to Super-EUSO (Fig. 15). 


\section{UHECR 2012}

\section{SUMMARY AND CONCLUSION}

We are children playing on the shoulder's of the three giants: John Linsley, Livio Scarsi, and Yoshiyuki Takahashi. Space-based observation of extensive airshowers is the next generation technology to open a new field of astronomy and astrophysics through charged particles and neutrinos at the highest energy above $10^{20} \mathrm{eV}$.

\section{References}

[1] Benson, R. and Linsley, J., 1981, Satellite observation of cosmic ray air showers, In International Cosmic Ray Conference, 17th, Paris, France, July 13-25, Conference Papers. Volume 8

[2] Takahashi, Y. 1995, Maximum-energy Auger Air Shower Satellite (MASS) for Observing Cosmic Rays in the Energy Region $10^{19-22} \mathrm{eV}$, Proceedings of 24th International Cosmic Ray Conference, Vol. 3, held August 28-September 8, 1995 in Rome, Italy. Edited by N. Iucci and E. Lamanna

[3] Linsley, J., Search for the end of the cosmic ray energy spectrum, 1998, Workshop on observing giant cosmic ray air showers from $>10^{20} \mathrm{eV}$ particles from space. AIP Conference Proceedings, Volume 433, pp. 1-21

[4] Streitmatter, R., 1998, Orbiting Wide-angle Light-collectors (OWL): Observing cosmic rays from space, Workshop on observing giant cosmic ray air showers from $>10^{20} \mathrm{eV}$ particles from space. AIP Conference Proceedings, Volume 433, pp. 95-107 (1998)

[5] Scarsi, L. et al. 2001, EUSO-Extreme Universe Space Observatory, Proceedings 27th ICRC, Hamburg, D, Vol. HE, pp.839-842

[6] Parmar, A. et al., 2003, The Extreme Universe Space Observatory (EUSO) Mission in the Context of ESA, Proceedings of the 28th International Cosmic Ray Conference. July 31-August 7, 2003. Trukuba, Japan. Under the auspices of the International Union of Pure and Applied Physics (IUPAP). Editors: T. Kajita, Y. Asaoka, A. Kawachi, Y. Matsubara and M. Sasaki, p.1073.

[7] EUSO Consortium, 2004, The EUSO Mission, Nuclear Physics B Proceedings Supplements, Volume 134, p. 15-22.

[8] Takahashi, Y., 2007, A giant natural TPC $(500 \mathrm{~km}) 3$ to observe extremely high energy cosmic particles-JEM EUSO telescope on International Space Station, Journal of Physics: Conference Series, Volume 65, Issue 1, pp. 012022

[9] JEM-EUSO Collaboration, 2007, The JEM-EUSO Mission, Proceedings of the 30th International Cosmic Ray Conference. July 3-11, 2007, Mérida, Yucatán, Mexico. Edited by Rogelio Caballero, Juan Carlos D’Olivo, Gustavo Medina-Tanco, Lukas Nellen, Federico A. Sánchez, José F. Valdés-Galicia. Universidad Nacional Autónoma de México, Mexico City, Mexico, 2008. Volume 5, p.1045-1048

[10] Takahashi, Y., JEM EUSO mission on the International Space Station, Journal of Physics: Conference Series, Volume 120, Issue 6, pp. 062013 (2008)

[11] Ebisuzaki, T. et al. 2010, The JEM-EUSO mission, 38th COSPAR Scientific Assembly. Held 18-15 July 2010, in Bremen, Germany, Symposium E, session 18, paper number E18-0030-10

[12] Ebisuzaki, T. et al., 2011, The JEM-EUSO mission, Proceedings of the 32nd International Cosmic Ray Conference (ICRC2011), held 11-18 August, 2011 in Beijing, China. Vol. 3 HE1.4: Extensive Air Showers and HE Cosmic Rays., p. 10

[13] Alexandrov, V. V., et al. 2001, Space Experiment "TUS" For Study Of Ultra High Energy Cosmic Rays, Proceedings of the 27th International Cosmic Ray Conference. 07-15 August, 2001. Hamburg, Germany. Under the auspices of the International Union of Pure and Applied Physics (IUPAP), p. 831

[14] Cordero, A., et al. 2005, Status of the Space Experiment TUS for UHECR Study, Proceedings of the 29th International Cosmic Ray Conference. August 3-10, 2005, Pune, India. Edited by 
B. Sripathi Acharya, Sunil Gupta, P. Jagadeesan, Atul Jain, S. Karthikeyan, Samuel Morris, and Suresh Tonwar. Mumbai: Tata Institute of Fundamental Research, 2005. Volume 8, p. 263

[15] Garipov, G. for Tus Team, 2006, The upgraded TUS space fluorescence detector for study of UHECR, 36th COSPAR Scientific Assembly. Held 16-23 July 2006, in Beijing, China. Meeting abstract from the CDROM, \#824

[16] Tkatchev, L., et al., 2007, Status of the TUS space detector preparation for UHECR study, Proceedings of the 30th International Cosmic Ray Conference. July 3-11, 2007, Mérida, Yucatán, Mexico. Edited by Rogelio Caballero, Juan Carlos D’Olivo, Gustavo Medina-Tanco, Lukas Nellen, Federico A. Sánchez, José F. Valdés-Galicia. Universidad Nacional Autónoma de México, Mexico City, Mexico, 2008. Volume 5, p. 873-876

[17] Tkachev, L, et al. 2011, The TUS Fresnel mirror production and optical parameters measurement., Proceedings of the 32nd International Cosmic Ray Conference (ICRC2011), held 11-18 August, 2011 in Beijing, China. Vol. 3 HE1.4: Extensive Air Showers and HE Cosmic Rays., p. 120

[18] Khrenov, B., 2001, Space Program KOSMOTEPETL (project KLYPVE and TUS) for the study of extremely high energy cosmic rays, OBSERVING ULTRAHIGH ENERGY COSMIC RAYS FROM SPACE AND EARTH: International Workshop. AIP Conference Proceedings, Volume 566, pp. 57-75

[19] Khrenov, B. A. et al. 2004, KLYPVE/TUS space experiments for study of ultrahigh-energy cosmic rays, Physics of Atomic Nuclei, Vol. 67, p. 2058-2061

[20] Ebisuzaki, T. et al. 2008, TUS space detector as a pathfinder for the next generation space detectors, Proceedings of the 30th International Cosmic Ray Conference. July 3-11, 2007, Mérida, Yucatán, Mexico. Edited by Rogelio Caballero, Juan Carlos D’Olivo, Gustavo MedinaTanco, Lukas Nellen, Federico A. Sánchez, José F. Valdés-Galicia. Universidad Nacional Autónoma de México, Mexico City, Mexico, 2008. Volume 5, p. 1089-1092

[21] Grossan, B., et al. 2011, Update on The Ultra-Fast Flash Observatory (UFFO) Pathfinder, GAMMA RAY BURSTS 2010. AIP Conference Proceedings, Volume 1358, pp. 419-422

[22] Petrolini, A., 2011, Ultra-High Energy Cosmic Particles studies from space: Super-EUSO, a possible next-generation experiment, Nuclear Instruments and Methods in Physics Research Section A, Volume 630, Issue 1, p. 131-135

[23] Takahashi, Y. and Ebisuzaki, T. 1998, Space Subaru: great science observatories in the space station ERA, Proc. SPIE Vol. 3356, p. 575-581, Space Telescopes and Instruments V, Pierre Y. Bely; James B. Breckinridge; Eds

[24] Ebisuzaki, T. and Takahashi, Y. et al. 2000, OWL-Orbital Wide-angle Light-collector for the air watch program, and multiple OWL, SPACE TECHNOLOGY AND APPLICATIONS INTERNATIONAL FORUM - 2000. AIP Conference Proceedings, Volume 504, pp. 279-280 